\title{
Measurement of the Parameters of Multiple Sinusoids Based on Binary Data
}

\author{
P. Carbone*, Fellow Member, IEEE, A. De Angelis*, Member, IEEE, F. Santoni*, A. Moschitta*, Member, IEEE \\ *University of Perugia - Engineering Department, via G. Duranti, 93 - 06125 Perugia Italy.
}

\begin{abstract}
This paper introduces a novel procedure for quick estimation of the parameters of a sum of sinusoidal signals based on one-bit measurements. Amplitude, phases and, frequencies of the signal components are assumed unknown, as well as the threshold level of the comparator used to produce measurement results. To provide enough information at the one-bit quantizer input, a sinewave is assumed to dither one of the two comparator's inputs. To ease the procedure's application, only the peak-to-peak amplitude of this dither signal is assumed known. Theoretical, simulation-based and experimental results validate the presented approach.
\end{abstract}

\section{INTRODUCTION}

Sums of sinusoidal signals having arbitrary frequencies are common signals in information-based systems. Besides being multi-purpose signals used in communication channels, they are used as test signals in measurement systems [1][3] and for system identification purposes [4]. They are employed to measure material properties in electrochemical impedance spectroscopy [5] and to characterize the stateof-health of rechargeable batteries [6]. One-bit quantization of sums of sinusoidal signals is a problem of interest in the area of compressive sensing [7], radar [8] and spectrum sensing applications [9], [10]. Thus, simplifying estimation of signal components in a sum of sinusoidal signals based on a one-bit quantized sequence is a problem of interest in the instrumentation and measurement community. It may result in more compact and simple measurement systems, in more easily deployable sensor networks and energy-aware Internetof-Things networks. At the same time, shifting complexity from the hardware to the signal processing domain may lead to simple integrated devices and applications.

Observe that, one-bit synthesis of multicomponent spectra is easily carried out through, e.g., maximum-length binary sequences (MLBS) [11]. Thus, a measurement chain made only of a single bit digital-to-analog (DAC) and analog-todigital converters (ADC) becomes possible. To account for the possible lack of information at the quantizer input due to binary representation of signals [12], quantization is assisted by a dither signal $[13]-[17]$. An alternative, well established one-bit measurement technique relies on $\Delta \Sigma$ ADCs that trade sampling rate and available bandwidth for equivalent

This research activity was partially funded through Identificazione e caratterizzazione accurata di sistemi e segnali, Ricerca di Base 2018 and 1-bit signal measurements, Ricerca di Base 2019 - University of Perugia. resolution. The method presented in this paper is based on equivalent-time sampling requiring the observation of several periods of the signal to be quantized and takes advantage of the periodicity of the input signal components. As a result, it does imply signal bandwidth limitations with respect to the used sampling rate.

This paper is an extension of the proceedings paper [18] that contains preliminary results, and shows how to quickly provide reasonably accurate estimate of sinusoidal components based on one-bit data, when the number of such components is known and by assuming sinusoidal dither. The procedure is described and detailed. The Cramér-Rao lower bound (CRLB) associated with this estimation problem is calculated. Theoretical, simulation and experimental results validating the measurement procedure are presented and discussed.

\section{A. Related Literature}

There is a vast research on the topic of parameter estimation and signal measurement based on one-bit data [8], [19]-[32]. The majority of published results rely on the assumption of Gaussian noise affecting the binary quantizer output. This assumption has general validity and may lead to mathematically tractable derivations. However, in the instrumentation and measurement domain, dither is intentionally added to improve measurement performance. When this occurs, Gaussian noise sources are less appealing than other sources, such as sinewaves. These latter signals can easily be generated even at high frequencies at a moderate or low power consumption.

The solution to this estimation problem belongs to a series of problems addressed in the literature using various approaches: while [8] assumes an ancillary sequence of known amplitude used before quantization to increase the amount of available information, [33] uses the expectation-maximization algorithm to process binary quantized data.

The technique presented in this paper has the advantage of not requiring knowledge of the exact values of the added dither sequence, but only of overall parameters such as the dither maximum amplitude. Moreover, by resorting to matrix inversion it proves to be a very fast method. It provides solutions that are very close to the optimal ones based on the maximum likelihood principle, analyzed in [28], [29] under the assumption of known frequency components. The measurement method aims at minimizing numerical calculations that are known to be time-consuming, in favor of ordi- 
nary matrix inversion methods that are efficiently performed even by a moderately complex microprocessor system. The method extends the binary quantile-based estimator (BQBE) published in [34] and is called in the following frequency $\mathrm{BQBE}$ (FBQBE). With respect to BQBE, FBQBE does not assume that the signal is periodic, nor that the component frequencies in the signal are known. Components can have arbitrary frequencies, although selectivity requirements imply a minimum difference to guarantee parametric estimation, as explained in the following sections.

\section{THE ANALYZED SYSTEM}

The system considered in this paper is shown in Fig. 1. In this Figure, the signal $s_{n}$ is given by the summation of a given number $K$ of sinewaves having amplitudes $A_{k}$, frequencies $\lambda_{k}$ and initial record phase $\phi_{k}$,

$$
\begin{aligned}
s_{n} & =\sum_{k=1}^{K} A_{k} \sin \left(2 \pi n \lambda_{i}+\phi_{k}\right) \\
& =\sum_{k=1}^{K} a_{k} \cos \left(2 \pi n \lambda_{k}\right)+b_{k} \sin \left(2 \pi n \lambda_{k}\right) \quad n=0, \ldots, N-1
\end{aligned}
$$

where $a_{k}=A_{k} \sin \left(\phi_{i}\right), b_{k}=A_{k} \cos \left(\phi_{i}\right), K$ is the number of signal components and $N$ the number of samples in the record. This signal is fed to a differential comparator, whose other input is connected to a zero-mean sinewave, $\eta_{n}$, having known amplitude. The comparator outputs a logical value of 1 if the difference $x_{n}-\eta_{n}$ is smaller than the value of an unknown threshold level $T$ and 0 otherwise. The added Gaussian noise source in Fig. 1 accounts for unmodeled noise affecting signals in the blockchain.

\section{A. Choice of the dither signal}

This signal chain models a very simple binary acquisition system, based on hardware possibly already available on a generic embedded platform, e.g. a microcontroller and a sinusoidal generator. The issue of evaluating the accuracy and precision that can be achieved in estimating the signal components becomes meaningful to enable new applications, based on a very limited amount of hardware resources. As a result, the choice of the dither signal becomes critical: a suitable choice of dither amplitude and probability density functions (PDFs) results in an optimal linearization of the otherwise strongly nonlinear behavior of the one-bit quantizer. While uniform dither exhibits optimal estimation properties, Gaussian noise still has desirable features [13]. However, the perfect realization of such uniform or Gaussian distributed signals either requires specific hardware or conflicts with the requirement of simplifying as much as possible the hardware needed for estimation purposes. For this reason, this paper considers sinusoidal dithering, that far from being optimal, can easily be generated even at very high frequencies with sufficiently accurate characteristics or at low frequencies with a current consumption in the order of tens of $\mu \mathrm{A}$ [35].

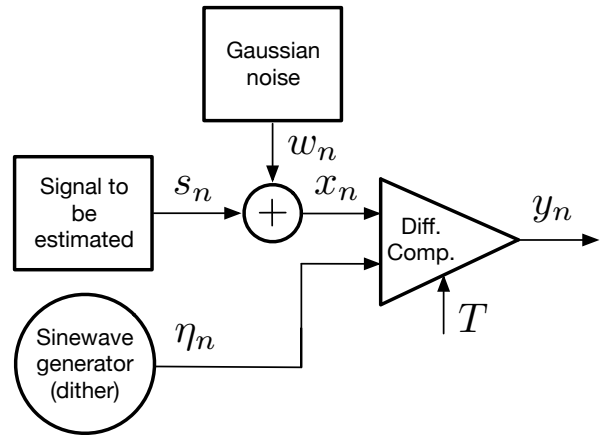

Fig. 1. Signal chain considered in this paper: the parameters in the generated signal are estimated based on the sequence at the binary output of the differential comparator, having threshold $T$, and whose other input is connected to sinusoidal dither source. Un-modeled Gaussian noise affects one of the comparator's inputs.

\section{The Main Estimation Procedure}

\section{A. Estimation of Signal Components' Parameters}

At first, define the two vectors of components to be esti-

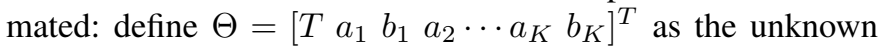
vector containing the comparator's threshold $T$ and the signal components amplitudes. Also define $\Lambda=\left[\lambda_{1} \cdots \lambda_{K}\right]^{T}$ as the vector of unknown frequency components, with ${ }^{T}$ as the transpose operator. The estimation procedure uses a modified version of the BQBE described in [34], based on the assumption of known component frequencies, and described in the following to ease readability. It will be explained in the case of a single signal component, i.e. by assuming $K=1$ in (1). All simplifying assumptions will be removed one by one in the following sections.

1) Simplified Signal Model : Neglecting the effect of Gaussian noise, assume the unquantized signal $x_{n}$, to be only composed by a single zero-mean sinusoidal component and thus to be expressed by:

$$
x_{n}=a_{1} \cos (2 \pi n \lambda)+b_{1} \sin (2 \pi n \lambda) \quad n=0, \ldots, N-1
$$

so that its quantized version, output by the differential comparator, is expressed by:

$$
y_{n}=Q\left(x_{n}-\eta_{n}\right) \quad n=0, \ldots, N-1
$$

where $Q(\cdot)$ returns 1 if its argument is below $T$ and 0 otherwise and where $\eta_{n}$ is the outcome of a sequence of random variables, all with known and invertible cumulative distribution function (CDF), $F_{\eta}(\cdot)$, having independent outcomes. Thus,

$$
p_{n} \triangleq P\left(y_{n}=1\right)=P\left(x_{n}-\eta_{n} \leq T\right)=F_{\eta}\left(T-x_{n}\right)
$$

where $P(\cdot)$ is the probability measure of the event at its argument and where it is assumed that $-\eta_{n}$ has the same CDF as $\eta_{n}$. Therefore, by using the inverse CFD (ICDF) $F_{\eta}^{-1}(\cdot)$ in (4) we obtain a linearization of the relationship between observed quantities and parameters to be estimated [19], [20]:

$$
F_{\eta}^{-1}\left(p_{n}\right)=T-x_{n}
$$


As shown in section III-A2 an estimator $\hat{p}_{n}$ of $p_{n}$ can be derived. If this value is substituted in (5) we obtain $F_{\eta}^{-1}\left(\hat{p}_{n}\right)=T-x_{n}$ that linearly relates the known quantity $F_{\eta}^{-1}\left(\hat{p}_{n}\right)$ to the unknown parameters $a_{1}$ and $b_{1}$ in $x_{n}$. Thus, a linear observation model results than can be solved by matrix inversion, considering the sequence of $n$ signal samples.

2) Estimating The Amplitude Components of the Simplified Signal Model: The estimation algorithm starts by assuming $\lambda$ known and by evaluating the equivalent sampling instants in (2) as $\lambda_{n}=\langle\lambda n\rangle$ for each value of $n$, where $\langle\cdot\rangle$ is the fractional part operator. The indices $\lambda_{n}$ are partitioned in $E>$ 0 subsets $\mathcal{I}_{j}$, such that

$$
\mathcal{I}_{j}=\left\{n \mid j \epsilon \leq \lambda_{n}<(j+1) \epsilon\right\}, \quad j=1, \ldots, E
$$

where $\epsilon=\frac{1}{E}$. If signal sampling is synchronous coherent, $\lambda$ is a rational number and $\mathcal{I}_{j}$ contains indices all resulting in the same value of $\lambda_{n}$, if $\epsilon$ is sufficiently small. Conversely, if signal sampling is asynchronous, $\lambda$ is an irrational number and each subset $\mathcal{I}_{j}$ contains indices resulting in different values of $\lambda_{n}$. However, if $\epsilon$ is sufficiently small, values of $\lambda_{n}$ in $\mathcal{I}_{j}$ are sufficiently close to each other to result in signal samples that can be considered approximately equal.

For each $j=1, \ldots, E$ the following is then calculated [34]:

$$
\hat{p}_{j}=\frac{1}{\left|\mathcal{I}_{j}\right|} \sum_{n \in \mathcal{I}_{j}} y_{n}
$$$$
z_{j}=F_{\eta}^{-1}\left(\hat{p}_{j}\right)
$$

$R_{j}=\left[1-\frac{1}{\left|\mathcal{I}_{j}\right|} \sum_{n \in \mathcal{I}_{j}} \cos \left(2 \pi \lambda_{n}\right)-\frac{1}{\left|\mathcal{I}_{j}\right|} \sum_{n \in \mathcal{I}_{j}} \sin \left(2 \pi \lambda_{n}\right)\right]$

where $\left|\mathcal{I}_{j}\right|$ is the cardinality of $\mathcal{I}_{j}$, and $R_{j}$ is a $1 \times 3$ row vector. Notice that if $\hat{p}_{j}=0$ or 1 , the ICDF might not be defined and the whole set of values in (7) is discarded from further processing. Then, the following three vectors/matrices are defined:

$$
Z=\left[\begin{array}{c}
z_{1} \\
z_{2} \\
\vdots \\
z_{L}
\end{array}\right] \quad H=\left[\begin{array}{c}
R_{1} \\
R_{2} \\
\vdots \\
R_{L}
\end{array}\right] \quad \Theta=\left[\begin{array}{c}
T \\
a \\
b
\end{array}\right]
$$

where $L \leq E$ and where $L$ is equal to $E$ when none of the elements in (7) are discarded, that is when $0<p_{j}<1$ for all values of $j=1, \ldots, E$. In (8), $Z$ is $L \times 1, H$ is $L \times 3$ and $\Theta$ is $3 \times 1$. By observing that the linearized model can be written as $Z=H \Theta$, where both $Z$ and $H$ are obtained by processing the measured one-bit data, an estimate of $\Theta$ is obtained by [36]:

$$
\hat{\Theta}=\left(H^{T} H\right)^{-1} H^{T} Z .
$$

Observe that the value of $\epsilon$ controls the tradeoff between the estimator bias and variance [34]. In fact, a very small value of $\epsilon$ guarantees a reduced bias because indices in $\mathcal{I}_{j}$ in 6 refer to very similar values of $\lambda_{n}$. However, when $\epsilon$ is a small value, intervals $\mathcal{I}_{j}$ will each contain few samples, resulting in a large variance of the random quantities defined in (7). Given that the number of such intervals is $E=\frac{1}{\epsilon}$, the average number of samples associated with each interval decreases, increasing the overall estimator variance. Conversely, when $\epsilon$ increases, estimation variance is reduced at the price of a larger bias. As indicated in [34], the choice $\epsilon=\frac{1}{\sqrt{N}}$ provides reasonable results.

\section{B. Estimation of all Signal Parameters}

The estimation of all $K$ components in the signal is done by estimating each component one-at-a-time using the method described in Sect. III-A2 In fact, since $s_{n}$ contains sinewaves having unrelated frequencies, the BQBE cannot be applied as is, because it requires periodicity of the parametric sequence. However, it can be used on single sinusoidal components considered one-at-a-time if their frequencies are assumed known. This implies that when the $k$-th component is estimated, all other components with indices different from $k$ are considered as disturbances. Observe that sinusoidal and cosinusoidal components having different frequencies are orthogonal [36]. Thus, the BQBE estimator tends to cancel the effect of unmodeled signal components when it is used to estimate the sinewaves one-at-a-time. In fact, as shown in [34], the matrix $\left(H^{T} H\right)^{-1}$ in $\sqrt{9}$ is approximately diagonal and, as shown by $(8), H^{T}$ contains the outcomes of sampled cosine and sine waves having approximately the same frequency $\lambda$. Thus, when $\left(H^{T} H\right)^{-1} H^{T}$ is multiplied by $Z$, the effects of all components having a frequency different from $\lambda$ tend to vanish.

An additional requirement for the application of the $\mathrm{BQBE}$ is that the sinusoidal frequency must be known. For each sinewave component, an initial estimate of the signal frequency is obtained by selecting the frequency of the discretetime Fourier transform (DFT) of the one-bit quantized signal having the largest magnitude. Refinement of the estimation is done by running a maximum likelihood estimator.

Thus, the following procedure provides the estimates of the signal parameters for $k=1, \ldots, K$, where steps 2)-4) are iterated until completion. The four steps are followed for each of the $K$ single signal components:

1) initial estimate of component frequency: for a given value of $k$, the DFT of $y_{n}$ is considered and an initial estimate $\hat{\lambda}_{k}$ of $\lambda_{k}$ is obtained. In case the largest peak is associated with the added dither signal, the corresponding samples are first removed from the DFT. Because of the severe one-bit amplitude quantization the output signal spectrum is heavily affected by large narrow-band spectrum components that may be larger than the selected component. In this case, knowledge of approximate values of the component frequencies can results in initial estimates for the iterative procedure described in the following;

2) estimation of the amplitude and phase of the selected signal component and of the quantizer threshold: the amplitudes $a_{k}$ and $b_{k}$ of the selected $k$-th component 
and the quantizer threshold $T$ are estimated by using the BQBE through (9);

3) iterative refinement of the estimated component frequency: the likelihood function is approximated on the basis of the calculated parameters and the frequency value $\hat{\lambda}_{k}$ estimated in 1) by the DFT. The frequency estimate is adjusted repeatedly until a maximum is obtained via numerical processing. By assuming the dither to have independent outcomes, and thus by neglecting sample correlation, given the binary nature of each sample in $y_{n}$, the estimated log-likelihood function $\hat{L}(Y ; \Theta)$, is

$$
\begin{aligned}
\hat{L}(Y ; \Theta)= & \sum_{n=0}^{N-1} y_{n} \log \left(F_{\eta}\left(\hat{T}-\hat{s}_{n}\right)\right) \\
& +\left(1-y_{n}\right) \log \left(1-F_{\eta}\left(\hat{T}-\hat{s}_{n}\right)\right),
\end{aligned}
$$

where $Y=\left[\begin{array}{llll}y_{0} & y_{2} & \cdots & y_{N-1}\end{array}\right]^{T}$ is the $N \times 1$ vector containing the binary quantizer output sequence and $\hat{T}$ is the transition level estimated by the BQBE in step 2). In $\left[10,, \hat{s}_{n}\right.$ is the input sequence estimated in step 2) and evaluated as,

$\hat{s}_{n}=\hat{a}_{1} \cos (2 \pi \hat{\lambda} n)+\hat{b}_{1} \sin (2 \pi \hat{\lambda} n) \quad n=0, \ldots, N-1$

where $\hat{a}_{1}$ and $\hat{b}_{1}$ are estimated by the BQBE as in 9 and $\hat{\lambda}$ is the current value of the searched parameter maximizing the approximate log-likelihood function 10 . Thus step 2) and 3) are repeated until a maximum in (10) is obtained;

4) iteration over the $K$ signal components: once a maximum is reached in the previous step, the algorithm processes the next component. Accordingly, the samples in the DFT of $y_{n}$ associated with the component that was already processed are discarded and steps 1)-3) are repeated until all $K$ components are estimated.

Observe that numerical maximization is performed only to estimate the component frequencies one-at-a-time, while the comparator's transition level and the amplitude and phase of each component are estimated by matrix inversion, as shown by (9).

\section{Practical issues}

If frequency components are too close, the coarse search done by using the DFT in step 1) will fail to discriminate between them. Similarly, if the magnitudes of the components in (1) are too small, they might not be the largest ones in the DFT of the quantized signal. In fact, since one-bit quantization is a strongly nonlinear transformation it introduces severe harmonics [37] that may be confused with the components to be estimated. The FBQBE will still work properly if rough estimates of the component frequencies are known, e.g. when jitter affects otherwise known ratios of sampling frequency and components' frequencies.

Notice that because of the finite number of samples, the level of applicability of the orthogonality principle recalled in Sect. III-B will increase as $N$ increases. Thus, the robustness of the BQBE to the effect of unmodeled sinewaves other than the one currently estimated will be a function of $N$. Observe also that the BQBE will still provide reasonable estimates of the sinewave parameters even if a single signal period is recorded as long as the number of samples used to estimate $\hat{p}_{j}$ in (7) is sufficiently large. In the single-period case, this occurs when the signal is highly oversampled. Notice however that the frequency-selectivity property of the DFT used to roughly estimate the components' frequencies sets a lower-bound on the minimum number of sinewave periods needed to resolve the various signal components.

The FBQBE is presented here as a procedure processing finite data segments. samples dynamically. As an alternative, recurrent relations between sequential observation matrices and problem solution can be derived [36]. Stationarity of the parameters' values of the signal to be estimated remains however a requirement within the same data segment.

\section{Dither noise distribution}

The distribution of the dither sequence $\eta$ plays a significant role in determining the properties of the described estimator. Several articles suggest the usage of Gaussian noise, also because of the possibility to derive tractable mathematical expressions. However, the precise generation of Gaussian noise using a practical source embedded in a systems, introduces circuital requirements that are not easy to fulfil under processvoltage-temperature variations. Thus, the main idea of pushing processing from the hardware to the software domain, based on the usage of simple hardware to acquire a complex signal, would be impacted by the requirement of a non-trivial incircuit noise generator. As an alternative, this article considers as a dither source a sinewave, having random initial record phase. Consider that contrary to a Gaussian source with weakly-correlated outcomes, in this case, the dither samples are highly correlated. While this assumption is not taken into account by $(10)$, the described procedure is robust enough to still provide meaningful results. This is partly caused by the asynchronous sampling of a sinewave that results in a sequence of signal amplitudes that tend to appear uncorrelated and chaotic. Thus, if the user avoids synchronization of the acquired sinusoidal components with the added sinewave, the estimator will approximately work as expected. Under these assumptions, the CDF of a sinusoidal sinewave having a uniformly distributed random phase can be expressed as [38]:

$$
F_{\eta}(x)= \begin{cases}0 & x \leq a \\ \frac{2}{\pi} \arcsin \left(\sqrt{\frac{x-a}{b-a}}\right) & a<x<b \\ 1 & x \geq b\end{cases}
$$

where $a$ and $b$ represents the minimum and maximum values assumed by the random variable. Thus, for $0<p<1$, its inverse is given by:

$$
F_{\eta}^{-1}(p)=(b-a) \sin ^{2}\left(\frac{\pi}{2} p\right)+a .
$$




\section{E. Comparison with maximum likelihood estimation}

As an alternative estimation procedure, the maximum likelihood estimator (MLE) could be used. The MLE solves a convex problem when the signal frequency is known and the dither is a Gaussian-distributed random process [28], [29]. When this hypothesis applies, [34] shows that the BQBE provides solutions that are close to the MLE. This paper extends the BQBE to be applicable when a signal having several components with arbitrary frequencies is assumed, that is when the hypothesis of the problem convexity can not be invoked, and under the assumption of sinusoidal dither. Since the $\mathrm{BQBE}$ is based on matrix inversion, the FBQBE can be used as a quick alternative to a solution based on numerical data processing provided by the MLE, or as an MLE initializer. Moreover, the MLE requires tuning and optimization of the iterative numerical maximization algorithm that might incur a more complex operation than applying the FBQBE.

\section{F. Simulation Results}

Monte Carlo simulations were done to validate the FBQBE. Fifty data records with lengths $N=10^{3}, \ldots, 2 \cdot 10^{5}$ were processed to estimate the parameters in a $K=6$ components signal, whose values are shown in Tab. [1. assuming a comparator's threshold $T=0.0366 \mathrm{~V}$. The dither sequence was simulated as:

$$
\eta_{n}=\frac{b-a}{2} \sin \left(2 \pi \lambda_{d} n+\phi\right), \quad n=0, \ldots, N-1
$$

with $b=-a=0.6 \mathrm{~V}, \lambda_{d}=\pi / 200$ and $\phi$ as a random variable, uniformly distributed in $[0,2 \pi)$ in each Monte Carlo record. Independent, zero-mean Gaussian distributed noise with standard deviation equal to 0.03 was added before quantization to simulate the effect of an unmodeled noise source, $w_{n}$, as shown in Fig. 1. In each record, $\Theta$ and $\Lambda$ were estimated to provide mean values and mean square-errors (MSE), over the estimated parameters, shown in Fig. 2.4. In particular, the parameters in $\Lambda$ were estimated one-at-a-time as described in Sect. III-B, by using the fminsearch function in MATLAB.

Fig. 2 shows that all parameters are estimated with decreasing mean MSE, when $N$ increases. As expected, the estimation accuracy depends on the considered parameter: the frequency is estimated very accurately as the output sequence is very sensitive to deviation in the frequency values. Conversely, accuracy in the estimation of component phases is lower. Phase errors were calculated as $\angle e^{j \hat{\phi}_{k}-j \phi_{k}}$, where $\hat{\phi}_{k}$ and

TABLE I

SIMULATION PARAMETERS USED TO OBTAIN RESULTS SHOWN IN FIG. 24

\begin{tabular}{clll} 
Component & Frequency & Amplitude & Phase \\
\hline 1 & 0.11768123 & 0.4 & $\pi / 13$ \\
2 & 0.1712431234 & 0.4 & $-3 \pi / 41$ \\
3 & 0.3134314 & 0.32 & $-\pi / 15$ \\
4 & 0.4121324 & 0.32 & $5 \pi / 6$ \\
5 & 0.2524513 & 0.3 & $6 \pi / 7$ \\
6 & 0.467123 & 0.3 & $\pi$
\end{tabular}
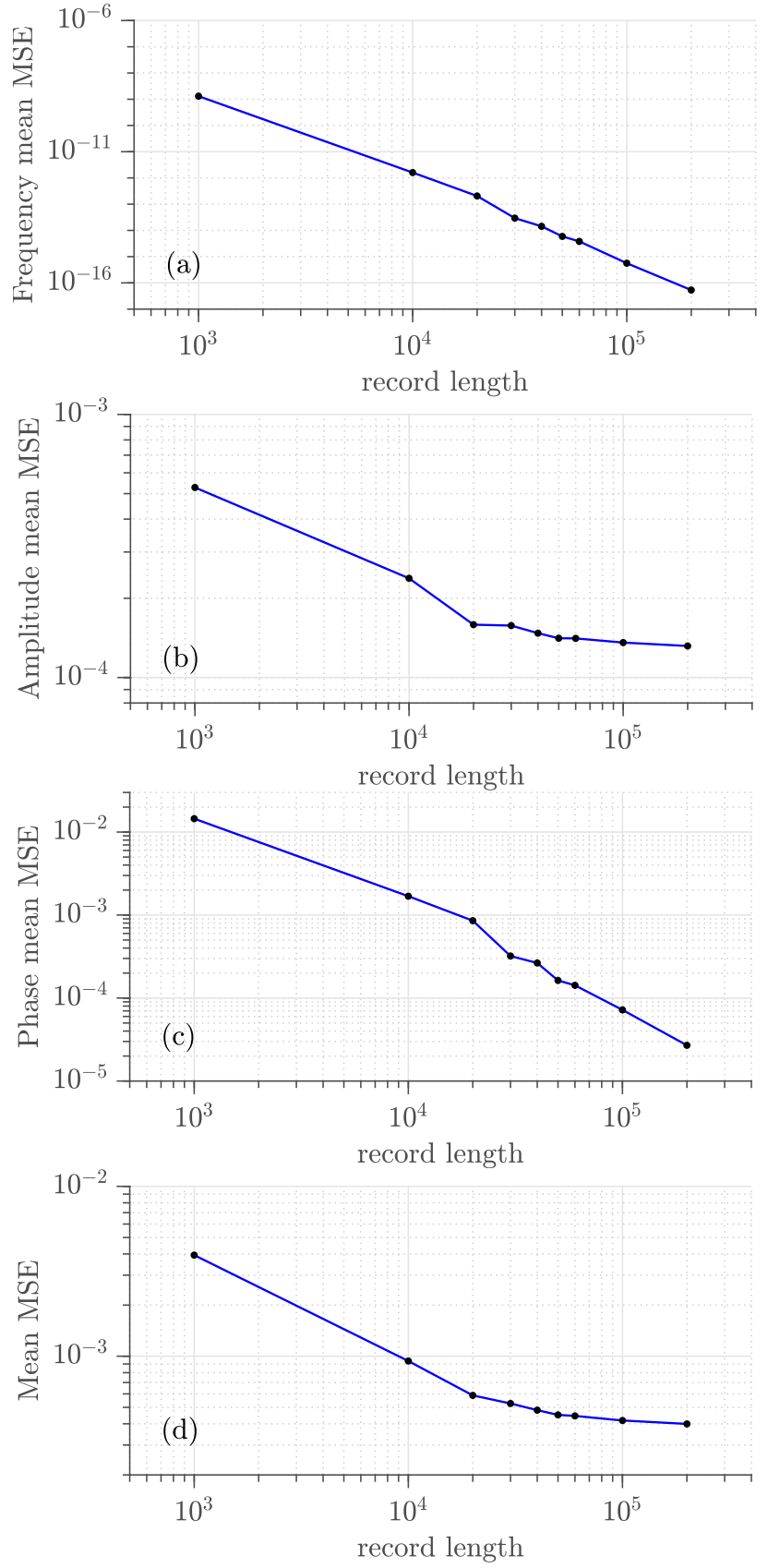

Fig. 2. Monte Carlo based simulation results: error performance of the FBQBE used to estimate the six sinusoidal components having parameters shown in Tab. I] as a function of the single record length. Shown is the mean MSEs associated with the estimation of the component frequencies (a), amplitudes (b), phases (c). Figure (d) shows the mean square error evaluated in the time-domain. Each point in graphs (a)-(c) is obtained by averaging 50 outcomes, each obtained by calculating the mean value of the six squared errors in the estimation of the parameters (frequency, amplitude, phase) of the six signal components. In each record, the dither signal had a random initial record phase. The graph in (d) shows the mean square-error between the reference and the reconstructed signal based on the usage of FBQBE.

$\phi_{k}, k=1, \ldots, 6$ are the estimated and true phase angles, respectively.

Overall average estimation times are shown in Fig. 3 


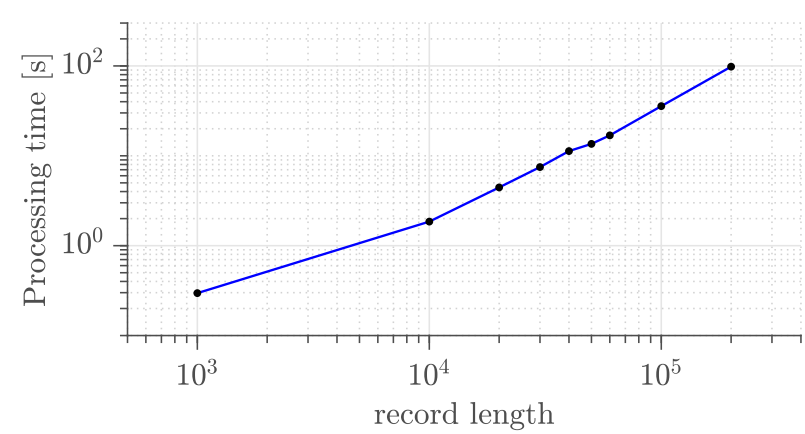

Fig. 3. Simulation results: processing time based on a 2-core I7 CPU, as a function of the record length when using the FBQBE applied to the same problem as in Fig. 2

Calculations were performed on a 2-core I7 CPU computer using a commercial mathematical simulation software and were thus not based on a fully optimized algorithm. Results show that the FBQBE quickly processes even moderately large records.

\section{EXPERIMENTAL RESULTS}

Experimental results were obtained to validate the FBQBE. A simple instruments was built using a PSOC 5LP prototyping board. This is a low-cost microcontroller that includes programmable analog parts to simply realize signal processing blocks. An internal comparator was used to generate the onebit sequence. An 8-bit DAC and 12-bit ADC were used to generate the sinusoidal dither signal and to acquire a fullresolution reference signal, respectively. On one input of the comparator was connected the sum of three sinusoidal signals. These were generated by an external commercial source generator with nominal parameters as shown in Tab. II A DC offset of $2.5 \mathrm{~V}$ was added to take into account the comparator input voltage span. On the other comparator input the internal DAC output was connected. A sinusoidal signal having frequency $952.381 \mathrm{~Hz}$, amplitude $0.4 \mathrm{~V}$, and DC offset $2.5 \mathrm{~V}$ was generated by the DAC. The one-bit comparator output, sampled at $100 \mathrm{ksamples} / \mathrm{s}$ was processed by the FBQBE to provide results shown in Fig. 5: Figure (a) shows the estimated signal and the reference signal, as returned by the internal 12-bit ADC. Figure (b) shows the estimation error. The estimated signal paramaters are shown in Tab. II Besides the bias associated with the FBQBE, other deviations from the nominal values may be due to uncertainties in the exact value of the internally generated dither signal, due to crosstalk between the lines connecting the comparator's inputs, as shown by an analysis of the spectrum of the reference signal. An additional explanation might be the finite dither DAC 8-bit resolution that limits the accuracy with which a PDF with perfect bathtub-like distribution is realized, when the corresponding $\mathrm{CDF}$ is given by (12). However, given the severe one-bit quantization, the FBQBE provides valid results for all application cases described in the introduction Section.
TABLE II

PARAMETERS' NOMINAL AND ESTIMATED VALUES USED TO OBTAIN EXPERIMENTAL RESULTS SHOWN IN FIG.5

\begin{tabular}{crl|ll}
$\begin{array}{c}\text { Component Frequency } \\
{[\mathrm{kHz}]}\end{array}$ & $\begin{array}{l}\text { Amplitude } \\
{[\mathrm{V}]}\end{array}$ & $\begin{array}{l}\text { Estimated } \\
\text { Frequency } \\
{[\mathrm{kHz}]}\end{array}$ & $\begin{array}{l}\text { Estimated } \\
\text { Amplitude } \\
{[\mathrm{V}]}\end{array}$ \\
\hline 1 & 3.123 & 0.12 & 3.121 & 0.135 \\
2 & 6.752 & 0.09 & 6.748 & 0.105 \\
3 & 16.481 & 0.19 & 16.470 & 0.213
\end{tabular}
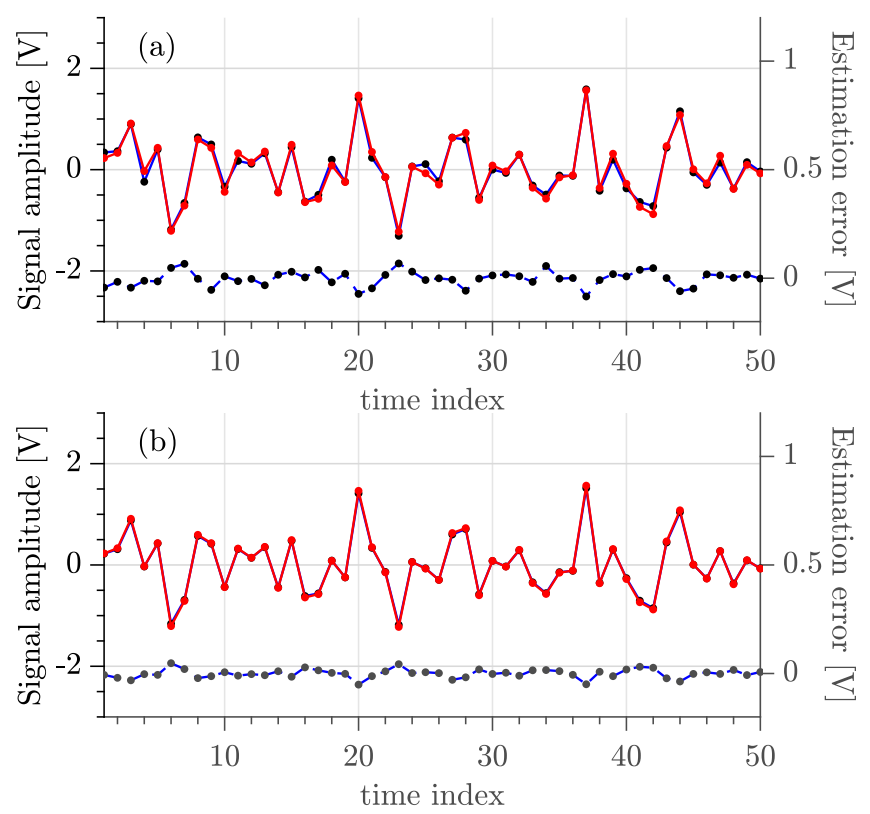

Fig. 4. Monte Carlo based simulation results based on 50 data records. Left axis: mean value of the original (black) and the reconstructed (red) signals when using the FBQBE based on $N=10^{3}$ (a) and $N=2 \cdot 10^{5}$ (b) samples, assuming the same simulation parameters as in Fig. 2 Right axis: the bottom graph in both figures shows the mean estimation error.

\section{Calculation of the Cramér-Rao Lower Bound}

In this section it will shown how to calculate the CramérRao Lower Bound (CRLB) associated with this estimation problem [36]. The CRLB provides a lower bound on the variance of any unbiased estimator of the searched parameter. Accordingly, define the noiseless version of the processed signal as in (1) and $\Theta=\left[\theta_{1} \cdots \theta_{p}\right]^{T}=$ $\left[\begin{array}{lllllllll}T & a_{1} & b_{1} & a_{2} & \cdots & a_{K} & b_{K} & \lambda_{1} \cdots & \lambda_{K}\end{array}\right]^{T}$ as the unknown vector containing the comparator's threshold $T$, the signal components amplitudes and the component frequencies $\lambda_{i}$.

Using a differential comparator and a dither sequence $\eta_{n}$ :

$$
y_{n}=Q\left(s_{n}(\Theta)+\eta_{n}\right) \quad n=0, \ldots, N-1
$$

where $Q(\cdot)$ returns 1 if its argument is not larger than $T$ and 0 otherwise and where $\eta_{n}$ is the outcome of a sequence of random variables, all with known and invertible $\mathrm{CDF}, F_{\eta}(\cdot)$. Thus,

$$
p_{n} \triangleq P\left(y_{n}=1\right)=P\left(s_{n}(\Theta)+\eta_{n} \leq T\right)=F_{\eta}\left(T-s_{n}(\Theta)\right)
$$



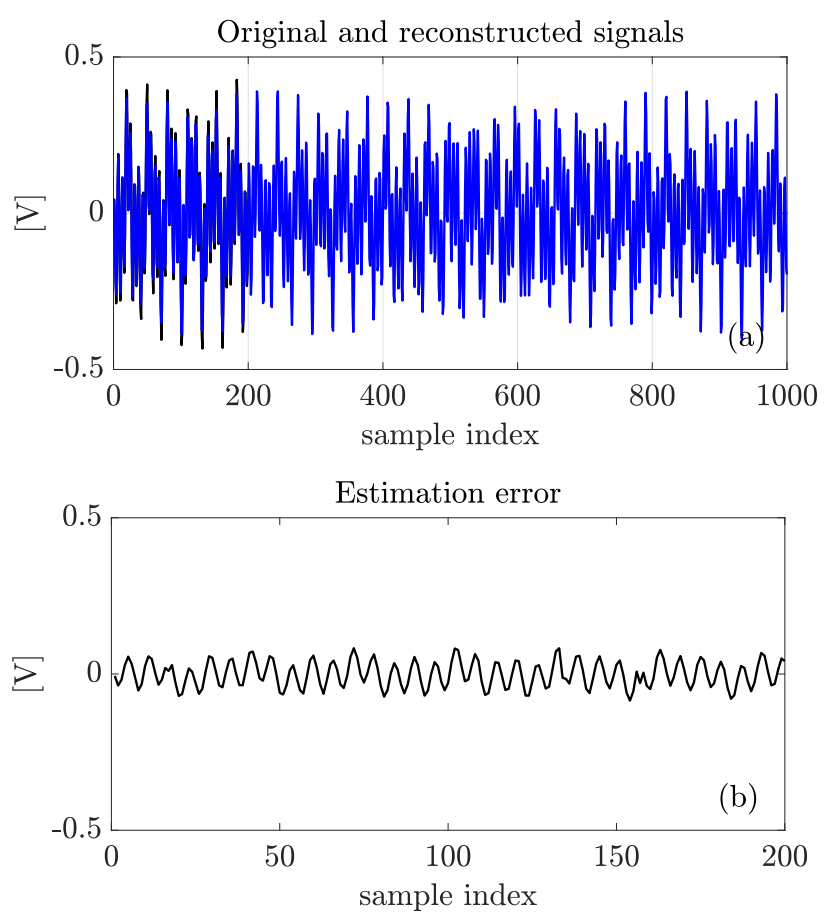

Fig. 5. Experimental results based on the FBQBE: estimation of a signal including three sinusoidal components having non-harmonic frequencies using one-bit quantized data. Sinusoidal dither with $0.8 \mathrm{~V}$ peak-to-peak amplitude was used. Figure (a) shows both the original (black) and the estimated signal (blue), while figure (b) shows the estimation error.

where $P(\cdot)$ is the probability measure of the event at its argument. Assume the dither signal as:

$$
\eta_{n}=A \cos (2 \pi \lambda n+\phi),
$$

where $\phi$ is a uniform random variable in $[0,2 \pi), \lambda$ is the normalized frequency and $A$ the maximum dither amplitude. Notice that independence of dither samples is not assumed in the following, contrary to the hypothesis made in Sect. III-A1. Because of the periodicity of the cosine function

$$
\begin{aligned}
\eta_{n} & =-A \cos (2 \pi \lambda n+\phi-\pi) \\
& =-A \cos \left(2 \pi\left(\langle\langle\lambda n\rangle+\bar{\phi}\rangle-\frac{1}{2}\right)\right)
\end{aligned}
$$

where $\bar{\phi}=\phi /(2 \pi)$ is uniform in $(0,1)$ and where the property $\langle\langle\alpha\rangle+\beta\rangle=\langle\alpha+\beta\rangle$ is used. Thus the event, $s_{n}(\Theta)+\eta_{n} \leq T$ results in

$$
-A \cos \left(2 \pi\left(\langle\langle\lambda n\rangle+\bar{\phi}\rangle-\frac{1}{2}\right)\right) \leq T-s_{n}(\Theta)
$$

that is

$$
\cos \left(2 \pi\left(\langle\langle\lambda n\rangle+\bar{\phi}\rangle-\frac{1}{2}\right)\right) \geq-\frac{T-s_{n}(\Theta)}{A}=\psi_{n}(\Theta)
$$

If $\left|\psi_{n}(\Theta)\right|>1$ the inequality in 21 is either always true or false, regardless of the value of $\bar{\phi}$. Conversely, if $\left|\psi_{n}(\Theta)\right| \leq 1$, since the argument of the cosine function in (21) belongs to $[-\pi, \pi)$, we have:

$$
-\frac{\arccos \left(\psi_{n}(\Theta)\right)}{2 \pi}<\langle\langle\lambda n\rangle+\bar{\phi}\rangle-\frac{1}{2} \leq \frac{\arccos \left(\psi_{n}(\Theta)\right)}{2 \pi}
$$

Since

$$
\langle\langle\lambda n\rangle+\bar{\phi}\rangle= \begin{cases}\langle\lambda n\rangle+\bar{\phi} & \bar{\phi}<1-\langle\lambda n\rangle \\ \langle\lambda n\rangle+\bar{\phi}-1 & \bar{\phi} \geq 1-\langle\lambda n\rangle\end{cases}
$$

By defining $\alpha_{n}(\Theta)=\frac{\arccos \left(\psi_{n}(\Theta)\right)}{2 \pi}$ and

$$
\begin{aligned}
& l_{1}(n ; \Theta)=\max \left(-\alpha_{n}(\Theta)-\langle\lambda n\rangle+\frac{1}{2}, 0\right) \\
& r_{1}(n ; \Theta)=\min \left(\alpha_{n}(\Theta)-\langle\lambda n\rangle+\frac{1}{2}, 1\right) \\
& l_{2}(n ; \Theta)=\max \left(-\alpha_{n}(\Theta)-\langle\lambda n\rangle+\frac{3}{2}, 0\right) \\
& r_{2}(n ; \Theta)=\min \left(\alpha_{n}(\Theta)-\langle\lambda n\rangle+\frac{3}{2}, 1\right)
\end{aligned}
$$

we have that 21] is satisfied when

$$
\begin{aligned}
& l_{1}(n ; \Theta)<\bar{\phi} \leq r_{1}(n ; \Theta) \\
& l_{2}(n ; \Theta)<\bar{\phi} \leq r_{2}(n ; \Theta)
\end{aligned}
$$

where the maximum and minimum operators are introduced to take into account the constraint $0 \leq \bar{\phi}<1$. Observe that because of how these boundary values are constructed only two of them will belong to the interval $(0,1)$, the other two being either 0 or 1 . If $\lambda$ is an irrational number, for each value of $n$, two new boundary values are generated other than 0 and 1 , that differ from those associated with previous values of $n$.

An example of the regions defined by (24) and 25) is shown in Fig. 6, where the dependence of the interval boundary values from $n$ and $\theta$ was dropped to ease readability. This Figure shows that when the coordinates $\left(\alpha_{n}(\Theta), \bar{\phi}\right)$ describe a point in the shaded area, the quantizer output is equal to 1 . For a given $n, \alpha_{n}(\Theta)$ remains determined and, as this graphs show, the quantizer outputs 1 when $\bar{\phi}$ belongs to a region that is lower and upper bounded by 0 and 1 and that remains otherwise defined by two additional values at the intercepts at

TABLE III

DEFINITION OF REGIONS FOR $\bar{\phi}$ WHERE THE QUANTIZER OUTPUTS 1 FOR GIVEN VALUES OF $n, \lambda$ AND $\alpha_{n}(\Theta)$.

\begin{tabular}{c|c} 
& $\langle\lambda n\rangle \leq 0.5$ \\
\hline $\begin{array}{c}\alpha_{n}(\Theta)<\langle\lambda n\rangle \\
\alpha_{n}(\Theta) \geq\langle\lambda n\rangle\end{array}$ & $0<\bar{\phi} \leq r_{2}(n ; \Theta)$ and $l_{2}(n ; \Theta)<\bar{\phi}<1$ \\
\hline & $\langle\lambda n\rangle>0.5$ \\
\hline & $\begin{array}{c}l_{1}(n ; \Theta)<\bar{\phi} \leq r_{1}(n ; \Theta) \\
\alpha_{n}(\Theta)<\langle\lambda n\rangle\end{array}$ \\
$\alpha_{n}(\Theta) \geq\langle\lambda n\rangle$ & $0<\bar{\phi} \leq r_{2}(n ; \Theta)$ and $l_{2}(n ; \Theta)<\bar{\phi}<1$
\end{tabular}




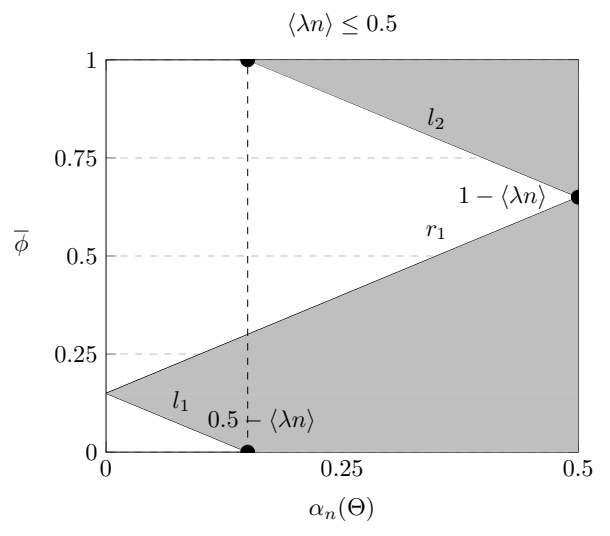

$\langle\lambda n\rangle>0.5$

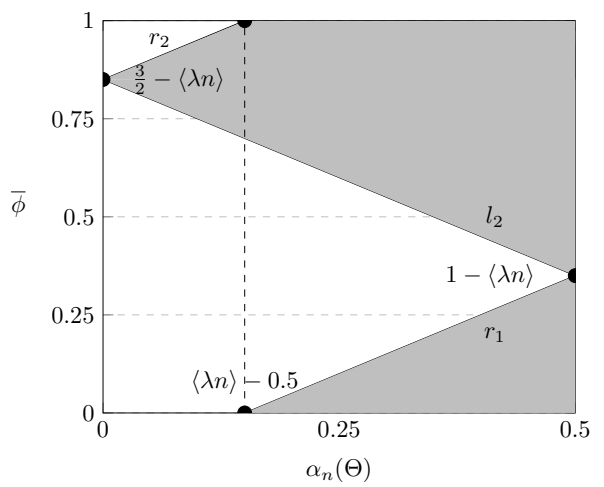

Fig. 6. Graphical representation of the inequalities defined in Tab. III with $\alpha_{n}(\Theta)=\frac{\arccos \left(\psi_{n}(\Theta)\right)}{2 \pi}, \psi_{n}(\Theta)=-\frac{T-s_{n}(\Theta)}{A}$ and $\bar{\phi}=\phi /(2 \pi)$. When the coordinates $\left(\alpha_{n}(\Theta), \bar{\phi}\right)$ describe a point in the shaded area, the quantizer output is equal to $1 ;\langle\lambda n\rangle=0.35$ is assumed in the upper graph and $\langle\lambda n\rangle=$ 0.65 is assumed in the lower graph.

the boundaries between shaded and unshaded areas. Equivalent information can be represented as in Tab. III.

Thus, when $n=0, \ldots, N-1$, a collection $\mathcal{C}$ of $M \leq 2 N+2$ such values, including the boundaries values 0 and 1 , is obtained. The interval $[0,1]$ is partitioned in subintervals, whose boundaries, $\alpha_{i}$, are defined by the ordered sequence $\mathcal{S}$ of the elements in the collection $\mathcal{C}$. At the same time, when $\bar{\phi}$ belongs to one of such subintervals, the quantizer output sequence obtained when $n=0, \ldots, N-1$ results in the same codeword of $N$ bits $B(\bar{\phi} ; \Theta)=\left[b_{i}(\bar{\phi} ; \Theta)\right]$ representing that particular subinterval. Observe that, because of how this mapping was constructed, different subintervals may result in the same codeword. Since $\bar{\phi}$ is uniformly distributed in $[0,1)$ the probability of $\bar{\phi}$ belonging to an interval defined by two consecutive values $\left[\gamma_{j}, \gamma_{j+1}\right], j=1, \ldots, M-1$ in $\mathcal{C}$ is equal to $\gamma_{j+1}-\gamma_{j}$, so that the full collection of probabilities is:

$$
\mathcal{P}_{\phi}=\left\{\gamma_{1}, \gamma_{2}-\gamma_{1}, \ldots, \gamma_{M}-\gamma_{M-1}, 1-\gamma_{M}\right\}
$$

When $n=0, \ldots, N-1$, the quantizer output results in an $N$-bit codeword, $B(\bar{\phi} ; \Theta)$ belonging to a collection $\mathcal{B}$ of unique codewords, with probability given by the sum of all probabilities in $\mathcal{P}_{\phi}$ corresponding to intervals resulting in that codeword $B(\bar{\phi} ; \Theta)$. Observe that since for every $n$ at most
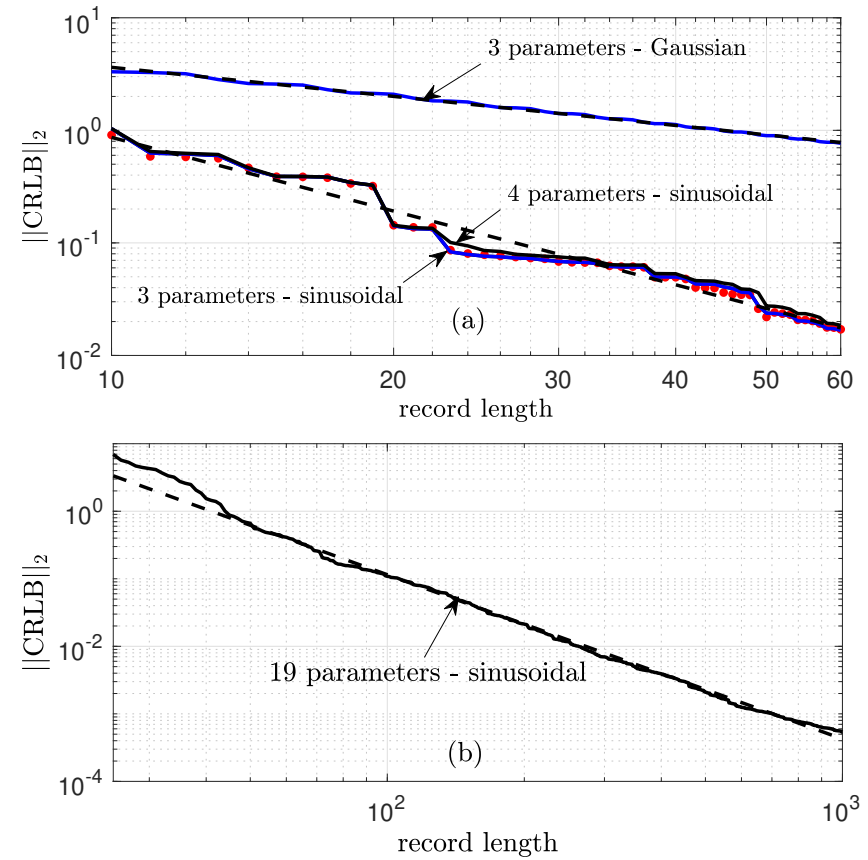

Fig. 7. Plot of the Euclidean norms of the Cramer-Rao Lower Bound associated with the estimation of $3-$ and 4 -parameters when $N=10, \ldots, 60$ (a). Results based on the theoretical derivations (solid lines) and on a Monte Carlo simulation (dots) based on $10^{7}$ records. The interpolating straight lines of the CRLB behavior, shown for both Gaussian and sinusoidal dither, have a slope of -0.86 and -2.17 ( 3 parameters) and -2.10 (4 parameters), respectively. (b) shows the norm when the parameters of six sinusoids and the threshold level are estimated. The interpolating rect line has a slope of -2.43 .

two values are added to the collection $\mathcal{C}$, the number of unique codewords $H<M \leq 2 N+2$, is much lower than the possible $2^{N}$ combinations of $N$ binary outputs.

\section{A. An example}

As an example consider $N=3$ and $s_{n}=A_{1} \sin \left(2 \pi n \lambda_{1}+\right.$ $\left.\phi_{1}\right)$ where $A_{1}=0.95, \lambda_{1}=3 / 17$ and $\phi_{1}=0.5$. Further assume $T=0$, and the dither signal parameter as $A=1, \lambda=\pi / 10$. Then, the values in $\mathcal{S}$ limited to four decimal points are:

$\mathcal{S}=\{0,0.0578,0.1350,0.2367,0.3253,0.6747,0.6856,1.0000\}$

Thus $M=2 N+2=8$. When $\phi$ is within any of the subintervals defined by values in $\mathcal{S}$, the $N$-bit codeword remains the same. The collection of codewords corresponding to all seven subintervals is:

$$
\mathcal{B}_{\phi}=\{[001],[000],[010],[000],[100],[001]\},
$$

with corresponding probability of occurrence when $\bar{\phi}$ is uniformly distributed in $[0,1)$ obtained by calculating differences of adjacent values in $\mathcal{S}$ to provide

$\mathcal{P}_{\phi}=\{0.0578,0.0772,0.1018,0.0885,0.3495,0.0109,0.3144\}$.

Since the two codewords 000 and 001 in $\mathcal{B}_{\phi}$ are repeated, the final collection of codewords $\mathcal{B}$ is obtained by selecting the $H=4$ unique codewords in $\mathcal{B}_{\phi}$. The probability of 
their occurrence is found by summing probabilities of events resulting in the same codeword:

$$
\mathcal{B}=\{[000],[001],[010],[100]\},
$$

with corresponding probabilities,

$$
\mathcal{P}=\{0.1766,0.3722,0.1018,0.3494\} .
$$

Thus, the probability mass function (PMF) of the vector $\mathbf{y}=\left[\begin{array}{llll}y_{0} & y_{1} & \cdots & y_{N-1}\end{array}\right]$ representing the binary sequence of quantizer output when $n=0, \ldots, N-1$ is:

$$
p_{\mathbf{Y}}(\mathbf{y} ; \Theta)= \begin{cases}0.1766 & \mathbf{y}=[000] \\ 0.3722 & \mathbf{y}=[001] \\ 0.1018 & \mathbf{y}=[010] \\ 0.3494 & \mathbf{y}=[100] \\ 0 & \text { otherwise }\end{cases}
$$

\section{B. Cramér-Rao Lower Bound}

The probabilities in 26) allow the definition of a probability mass function and of the corresponding log-likelihood function:

$$
L(\mathbf{y} ; \Theta)=\sum_{\mathbf{h} \in \mathcal{B}} I(\{\mathbf{y}=\mathbf{h}\}) \ln p_{\mathbf{Y}}(\mathbf{y} ; \Theta)
$$

where $I(\mathcal{E})$ is the indicator function of the event $\mathcal{E}$, so that the element in row $i$, column $j$ of the Fisher information matrix, for $i=1, \ldots, p \quad j=1, \ldots, p$ becomes [36]:

$$
\begin{aligned}
{[\mathbf{I}(\Theta)]_{i j} } & =E\left[\frac{\partial L(\mathbf{y} ; \Theta)}{\partial \theta_{i}} \frac{\partial L(\mathbf{y} ; \Theta)}{\partial \theta_{j}}\right] \\
& =\sum_{\mathbf{y} \in \mathcal{B}} \frac{1}{p_{\mathbf{Y}}(\mathbf{y} ; \Theta)} \frac{\partial p_{\mathbf{Y}}(\mathbf{y} ; \Theta)}{\partial \theta_{i}} \frac{\partial p_{\mathbf{Y}}(\mathbf{y} ; \Theta)}{\partial \theta_{j}}
\end{aligned}
$$

where $p$ is the number of parameters $\theta_{i}$ in $\Theta$, and where the partial derivatives can be calculated by observing that probabilities in (26) are defined as differences of known expressions. Then, diagonal elements of the inverted Fisher information matrix provide lower bounds to the variance of any unbiased estimator.

\section{Simulation Results}

The validity of the calculations described in Section $\mathrm{V}$ was proved using Monte Carlo simulations to evaluate distributions and approximate derivatives using numerical calculations. At first, a three- and four -parameter problem were setup with $K=1$ and $\Theta=\left[\begin{array}{lll}A_{1} & \lambda_{k} & \phi_{k}\end{array}\right]^{T}$ or $\Theta=\left[\begin{array}{lll}A_{1} & \lambda_{k} & \phi_{k}\end{array}\right]^{T}$. In this case both Monte Carlo and results obtained with the direct procedure were evaluated. Then, the CRLB was evaluated by the direct method in the case of the 19-parameters associated with the estimation of the three parameters of each of the six components listed in Tab II, along with the threshold level. The Euclidean norm of the components of the values on the principal diagonal of the inverse Fisher information matrix are reported in Fig. 7. For comparison purposes, this Fig. 7 ( a) also shows the behavior of the Euclidean norm of the CRLB obtained when assuming zero-mean uncorrelated Gaussian dither noise with standard deviation equal to 1 ,
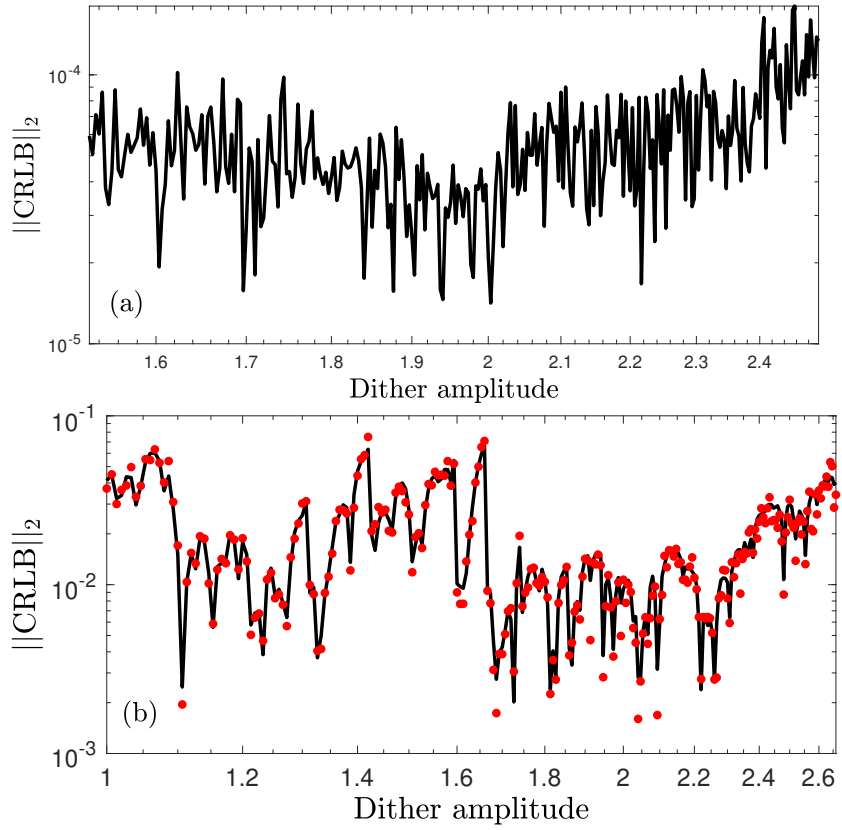

Fig. 8. Plot of the Euclidean norms of the Cramér-Rao Lower Bound associated with the estimation of the three parameters of a sinusoidal component with amplitude 2 , when $N=301$ (a), and $N=30$ (b) as a function of the dither amplitude. Results shown in (b) are based on the theoretical derivations (solid lines) and on a Monte Carlo simulation (dots) based on $9 \cdot 10^{6}$ records.

instead of sinusoidal dither [29]. It can be seen that the correlated noise sequences of the sinusoidal signal results in a quicker descent of the CRLB with the number of samples, than the uncorrelated Gaussian dither.

To appreciate the effect of the dither amplitude on the CRLB, the Euclidean norm of the components in the principal diagonal of the CRLB matrix are shown in Fig 8 in the case of $N=301$ (a) and $N=30$ (b), when assuming a single sinusoidal component with amplitude 2.0. In this latter case, also Monte Carlo plots are shown based on $9 \cdot 10^{6}$ records. Both graphs indicate a minimum when the dither amplitude approximately equals the signal amplitude. In fact, when the dither amplitude is lower than that, some of the sine wave samples are systematically under or over the quantizer threshold, irrespective of the dither magnitude. Conversely, increasing the dither amplitude beyond the signal amplitude only contributes to additional variance.

\section{CONCLUSION}

This paper describes a novel method to estimate the parameters of a multisine signal, having components with possibly unrelated frequencies, amplitudes, and phases, based on binary-only measurements. Since binary quantization is poorly informative, a sinusoidal dither signal is added before onebit quantization. Knowledge of the sinewave amplitude alone is sufficient to estimate all components' parameters using numerical maximization over a single parameter and simple matrix inversion. Simulation results prove the method's effectiveness by processing one-bit data to estimate the parameters 
in a signal having six sinusoidal components with unrelated frequencies. Experimental results show its effectiveness when processing one-bit data obtained by quantizing the sum of three sinusoids. Finally, extensive calculations are presented to derive the Cramér-Rao lower bound for this estimation problem.

\section{REFERENCES}

[1] "IEEE standard for terminology and test methods for analog-to-digital converters." [Online]. Available: https://doi.org/10.1109/ieeestd.2011. 5692956

[2] N. Fu, Z. Wei, L. Qiao, and Z. Yan, "Short-observation measurement of multiple sinusoids with multichannel sub-nyquist sampling," IEEE Transactions on Instrumentation and Measurement, Jan. 2020.

[3] S. Tomar and P. Sumathi, "Amplitude and frequency estimation of exponentially decaying sinusoids," IEEE Transactions on Instrumentation and Measurement, vol. 67, no. 1, pp. 229-237, Jan. 2018.

[4] R. Pintelon and J. Schoukens, System Identification: A Frequency Domain Approach. Wiley - IEEE Press, 2012.

[5] C.-L. Wei, Y.-W. Wang, and B.-D. Liu, "Wide-range filter-based sinusoidal wave synthesizer for electrochemical impedance spectroscopy measurements," IEEE Transactions on Biomedical Circuits and Systems, vol. 8, no. 3, pp. 442-450, Jun. 2014.

[6] J. Remmlinger, M. Buchholz, M. Meiler, P. Bernreuter, and K. Dietmayer, "State-of-health monitoring of lithium-ion batteries in electric vehicles by on-board internal resistance estimation," Journal of Power Sources, vol. 196, no. 12, pp. 5357-5363, Jun. 2011.

[7] K. Knudson, R. Saab, and R. Ward, "One-bit compressive sensing with norm estimation," IEEE Transactions on Information Theory, vol. 62 , no. 5, pp. 2748-2758, May 2016.

[8] J. Ren, T. Zhang, J. Li, and P. Stoica, "Sinusoidal parameter estimation from signed measurements via majorization-minimization based RELAX," IEEE Transactions on Signal Processing, vol. 67, no. 8, pp. 2173-2186, Apr. 2019.

[9] P. T. Boufounos and R. G. Baraniuk, "1-bit compressive sensing," in Proc. Conf. Inform. Science and Systems (CISS), Princeton, NJ, Mar. 19-21 2008.

[10] L. Jacques, J. N. Laska, P. T. Boufounos, and R. G. Baraniuk, "Robust 1bit compressive sensing via binary stable embeddings of sparse vectors," IEEE Trans. Inf. Theory, vol. 59, no. 4, pp. 2082-2102, Apr. 2013.

[11] A. H. Tan and K. R. Godfrey, Industrial Process Identification. Springer International Publishing, 2019.

[12] A. Moschitta, J. Schoukens, and P. Carbone, "Information and statistical efficiency when quantizing noisy DC values," IEEE Trans. Instrum. Meas., vol. 64, no. 2, pp. 308-317, Feb. 2015.

[13] R. Gray and D. Neuhoff, "Quantization," IEEE Transactions on Information Theory, vol. 44, no. 6, pp. 2325-2383, 1998.

[14] B. Widrow and I. Kollar, Quantization noise: Roundoff error in digital computation, signal processing, control, and communications. Cambridge University Press, 2008.

[15] P. Carbone, "Quantitative criteria for the design of dither-based quantizing systems," IEEE Transactions on Instrumentation and Measurement, vol. 46, no. 3, pp. 656-659, 1997.

[16] D. Rousseau and F. Chapeau-Blondeau, "Noise-improved bayesian estimation with arrays of one-bit quantizers," IEEE Transactions on Instrumentation and Measurement, vol. 56, no. 6, pp. 2658-2662, Dec. 2007.

[17] B. Ando, S. Baglio, S. Graziani, and N. Pitrone, "Measurements of parameters influencing the optimal noise level in stochastic systems," IEEE Transactions on Instrumentation and Measurement, vol. 49, no. 5, pp. 1137-1143, 2000.
[18] P. Carbone, A. D. Angelis, and A. Moschitta, "One-bit measurement of the parameters of multiple sinusoids with unknown frequency," in 2020 IEEE International Instrumentation and Measurement Technology Conference (I2MTC). IEEE, May 2020.

[19] A. Agresti, Categorical Data Analysis. Hoboken, N.J: WileyInterscience, 1996.

[20] L. Y. Wang, G. G. Yin, J.-F. Zhang, and Y. Zhao, System Identification with Quantized Observations. Birkhäuser Boston, 2010. [Online]. Available: https://doi.org/10.1007/978-0-8176-4956-2

[21] L. Y. Wang, J.-F. Znang, and G. Yin, "System identincation using binary sensors," IEEE Trans. Autom. Control, vol. 48, no. 11, pp. 1892-1907, Nov. 2003.

[22] L. Y. Wang and G. G. Yin, "Asymptotically efficient parameter estimation using quantized output observations," Autom., vol. 43, no. 7, pp. 1178-1191, July 2007.

[23] Y. Zhao, L. Y. Wang, G. G. Yin, and J.-F. Zhang, "Identification of Wiener systems with binary-valued output observations," Autom., vol. 43, no. 10, pp. 1752-1765, Oct. 2007.

[24] L. Y. Wang, G. G. Yin, Y. Zhao, and J.-F. Zhang, "Identification input design for consistent parameter estimation of linear systems with binaryvalued output observations," IEEE Trans. Autom. Control, vol. 53, no. 4, pp. 867-880, May 2008.

[25] P. Carbone, J. Schoukens, and A. Moschitta, "Dynamic signal measurements based on quantized data," IEEE Trans. Instrum. Meas., vol. 66, no. 2, pp. 223-233, Feb. 2017.

[26] G. Bottegal, H. Hjalmarsson, and G. Pillonetto, "A new kernel-based approach to system identification with quantized output data," Autom. vol. 85 , pp. 145-152, Nov. 2017.

[27] M. S. Stein, S. Bar, J. A. Nossek, and J. Tabrikian, "Performance analysis for channel estimation with 1-bit ADC and unknown quantization threshold," IEEE Trans. Signal Process., vol. 66, no. 10, pp. 2557-2571, May 2018.

[28] A. Ribeiro and G.B. Giannakis, "Bandwidth-constrained distributed estimation for wireless sensor networks-part i: Gaussian case," IEEE Trans. Signal Process., vol. 54, no. 3, pp. 1131-1143, Mar. 2006.

[29] A. Ribeiro and G. Giannakis, "Bandwidth-constrained distributed estimation for wireless sensor networks-part II: unknown probability density function," IEEE Trans. Signal Process., vol. 54, no. 7, pp. 2784-2796, July 2006.

[30] C. Gianelli, L. Xu, J. Li, and P. Stoica, "One-bit compressive sampling with time-varying thresholds: Maximum likelihood and the CramérRao bound," in 2016 50th Asilomar Conf. on Signals, Systems and Computers. IEEE, Nov. 2016.

[31] Christopher Gianelli and Luzhou Xu and Jian Li and Petre Stoica, "Onebit compressive sampling with time-varying thresholds for multiple sinusoids," in 2017 IEEE 7th Intern. Workshop on Computat. Advances in Multi-Sensor Adaptive Processing (CAMSAP). IEEE, Dec. 2017.

[32] A. Høst-Madsen and P. Handel, "Effects of sampling and quantization on single-tone frequency estimation," IEEE Trans. Signal Process., vol. 48 , no. 3, pp. 650-662, Mar. 2000.

[33] B. I. Godoy, G. C. Goodwin, J. C. Agüero, D. Marelli, and T. Wigren, "On identification of FIR systems having quantized output data," Autom. vol. 47, no. 9, pp. 1905-1915, Sep. 2011.

[34] P. Carbone, J. Schoukens, and A. Moschitta, "Quick estimation of periodic signal parameters from 1-bit measurements," IEEE Transactions on Instrumentation and Measurement, pp. 1-15, 2019.

[35] P. Karantzalis, A. Schultz, and C. Chang, "Lower power op amp: Low noise reference, utility sine wave," Available at https://www.analog.com/en/technical-articles/ lower-power-op-amp-low-noise-reference-utility-sine-wave.html

[36] S. Kay, Fundamentals of statistical signal processing. Englewood Cliffs, N.J: Prentice-Hall PTR, 1993.

[37] W. R. Bennett, "Spectra of quantized signals," Bell System Technical Journal, vol. 27, no. 3, pp. 446-472, Jul. 1948.

[38] A. Stuart, Kendall's Advanced Theory of Statistics, Distribution Theory. Wiley, feb 2010. 